\title{
The Relevance of Including Future Healthcare Costs in Cost-Effectiveness Threshold Calculations for the UK NHS
}

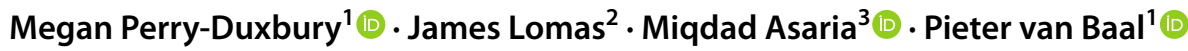

Accepted: 9 September 2021 / Published online: 26 October 2021

(c) The Author(s) 2021

\begin{abstract}
Background and Objective The supply-side threshold for the UK National Health Service has been empirically estimated as the marginal returns to healthcare spending on health outcomes. These estimates implicitly exclude future healthcare costs, which is inconsistent with the objective of making the most efficient use of healthcare resources. This paper illustrates how empirical estimates of the threshold within healthcare can be adjusted to account for future healthcare costs.

Methods Using cause-deleted life tables and previous work on future costs in England and Wales, we illustrate how such estimates can be adjusted.

Results While the effect of including future healthcare costs can have substantial effects on incremental cost-effectiveness ratios of specific life-extending interventions, we find that including future costs has relatively little impact (an increase of $£ 743$ per quality-adjusted life-year) on the threshold estimate.

Conclusions For some life-extending interventions the impact of including future costs on whether an intervention is deemed cost effective may be considerable.
\end{abstract}

\section{Key Points for Decision Makers}

This study illustrates, using cause-deleted life-tables, how empirical estimates of the threshold within healthcare can be adjusted to account for future healthcare costs.

The impact of including future costs on threshold estimates is limited in comparison to the impact of including future costs on the incremental cost-effectiveness ratio.

Including future medical costs in incremental costeffectiveness ratios is a necessary but not a sufficient condition for optimal decision making; the supply-side threshold should also reflect these costs.

Megan Perry-Duxbury

perryduxbury@eshpm.eur.nl

1 Erasmus School of Health Policy \& Management, Erasmus University Rotterdam, P.O. Box 1738, 3000 Rotterdam, DR, The Netherlands

2 Centre for Health Economics, University of York, York, UK

3 LSE Health, London School of Economics, London, UK

\section{Introduction}

A key criterion for deciding whether to reimburse a healthcare intervention is whether the estimated incremental costeffective ratio (ICER) lies below the relevant cost-effectiveness threshold [1]. In England and Wales, the National Institute for Health and Care Excellence (NICE) makes explicit use of such a threshold [2]. The NICE 2013 guidelines state that: 'A technology can be considered to be cost effective if its health benefits are greater than the opportunity costs of programmes displaced to fund the new technology, in the context of a fixed NHS budget' [3]. However, the current threshold used by NICE, which ranges from $£ 20,000$ to $£ 30,000$ per quality-adjusted life-year (QALY), reflects values implied by previous decisions made by NICE rather than an explicit consideration of the evidence regarding the potential health foregone caused by implementing new health technologies [4].

In theory, estimating the health foregone of displaced care, also referred to as the supply-side or $k$ threshold [5] when expressed per life-year (LY) or QALY, requires us to know precisely which activities would be displaced at the margin and estimate the health benefits forgone in displacing these activities. These thresholds could be estimated through (detailed) 'league tables', which rank interventions based 
on cost effectiveness. However, this would require knowledge regarding the cost effectiveness of all currently funded interventions. Additionally, we would need to assume that a new intervention would be funded by displacing the least cost-effective interventions on the list, which may not be what happens in practice. An alternative, empirically practical, approach is to estimate the cost per QALY of healthcare expenditure at the margin [5-9], which provides an estimate of the average cost effectiveness of unspecified interventions (potentially) displaced or expanded at the margin.

Interventions displaced or expanded at the margin may be quality of life improving, life extending or both. It has been argued that when an intervention has a life-extending element, the inclusion of future healthcare costs is necessary for a consistent estimate of cost effectiveness and an efficient allocation of the healthcare budget [10-14].

There are various arguments for the inclusion of future unrelated medical costs. First, quality-of-life and life expectancy estimates are usually obtained from people receiving unrelated care; it is inconsistent to exclude costs and include benefits of unrelated future medical care. [15] Second, given a fixed healthcare budget, increasing future unrelated medical costs via extending life leads to health opportunity costs. By excluding future unrelated medical costs, we underestimate the opportunity cost of these life-extending interventions. [12] Third, excluding future unrelated costs introduces a bias against interventions that do not add to future costs, namely, quality-of-life improving interventions. This paper takes the stance, for the above reasons, that future unrelated medical costs need to be included in economic evaluations.

Consider a patient who is treated for an otherwise fatal heart attack gains additional LYs and incurs additional related healthcare costs (e.g. cardiologist check-ups), but also incurs additional unrelated healthcare costs (e.g. cancer or dementia treatment). This is true not only for new treatments, but also for existing (and potentially replaced) treatments. Hence, estimates of the supply-side threshold should include both related and unrelated future healthcare costs. Existing analyses estimating these supply-side thresholds have ignored both related and unrelated future costs [7]. In this paper, we use the current most quoted supplyside threshold estimate for England and Wales [7] as the case to help describe how such empirical estimates of the supply-side threshold can be adjusted to account for future healthcare costs, and how this impacts the cost-effectiveness results of interventions when future costs are also included in an economic evaluation. There is some controversy surrounding this threshold estimate, regarding the assumptions made [16] and whether the use of a strict cost-effectiveness threshold is really necessary or helpful [17]. The aim of this paper is not to enter into these discussions, but to illustrate the importance of including future costs if such a supply-side threshold were to be implemented by NICE.
Future cost estimates were derived in earlier work [RW. ERROR - Unable to find reference:512] using aggregate data sources on National Health Service (NHS) spending by age, sex and time to death. Using mortality rates from England and Wales, survivor and decedent costs were estimated by age and sex. By combining these costs with survival curves of an intervention, the future unrelated medical costs (adjusted for double counting and related diseases) can then be added.

\section{Methods}

\subsection{Conceptual Model}

Assuming the aim of health maximisation and a fixed healthcare budget, an intervention can be implemented if its ICER is below the supply-side threshold, $k$. Both the ICER and $k$ can be viewed as a ratio of marginal costs to marginal benefits. For the ICER, the costs and benefits relate to a specific intervention for a known patient group (denoted with an asterisk $*$ ), while for $k$ (i.e. $\frac{\Delta \text { Costs }}{\Delta \text { QALYs }}$ ) they refer to a range and mix of interventions for various patient groups that are displaced at the margin:

$\frac{\Delta \text { Costs }^{*}}{\Delta \text { QALYs }^{*}}<\frac{\Delta \text { Costs }}{\Delta \text { QALYs }}$

Interventions that improve survival implicitly generate both future related and unrelated medical costs in LYs gained [10, 15, 18, 19]. By making a distinction between length of life and health spending conditional on being alive, we can rewrite the cost-effectiveness decision rule as:

$\frac{\Delta\left[L^{*} \times\left(c_{\mathrm{r}}^{*}+c_{\mathrm{u}}^{*}\right)\right]}{\Delta \text { QALYs }^{*}}<\frac{\Delta\left[L \times\left(c_{\mathrm{r}}+c_{\mathrm{u}}\right)\right]}{\Delta \mathrm{QALYs}}$

where $L$ denotes LYs and $c_{\mathrm{r}}$ and $c_{\mathrm{u}}$ are related and unrelated medical costs per year, respectively. All elements denoted with an asterisk $(*)$ relate to specific displaced interventions. Given that unrelated medical costs conditional on survival are independent of the intervention $\left(\Delta c_{\mathrm{u}}=0\right)$, Eq. 2 can be rewritten as:

$\frac{\Delta\left(L^{*} \times c_{\mathrm{r}}^{*}\right)+\Delta L^{*} \times c_{\mathrm{u}}^{*}}{\Delta \mathrm{QALYs}^{*}}<\frac{\Delta\left(L \times c_{\mathrm{r}}\right)+\Delta L \times c_{\mathrm{u}}}{\Delta \mathrm{QALYs}}$

The difference in unrelated costs between an intervention and comparator is solely dependent on the difference in LYs. Excluding future unrelated costs from estimations of $k$ and the ICER implicitly assumes that the incremental unrelated healthcare expenditures are zero or net out to zero, which seems unrealistic. Note also in this context that consistently excluding unrelated costs from both sides of Eq. (3) leads 
to an inefficient use of the healthcare budget and thus to health losses [12]. It is important to note here that the conceptual model for notational simplicity does not address the age dependency of related and unrelated medical costs, in our analyses, we took this into account.

\subsection{Updating the Threshold}

Claxton et al. [7] calculate the cost-effectiveness threshold using estimates of the effect of an annual $1 \%$ increase in the total healthcare budget on annual mortality rates. Expenditure and outcome elasticities were estimated using an instrumental variables approach. These elasticities were used to calculate the cost of a LY in each programme. The calculated LYs gained from the increase in the health budget were then adjusted for quality of life, taking account of sex, age and disease. The increase in total NHS spending divided by the sum of all QALY changes across all programme budgeting categories (PBCs) was presented as the relevant threshold. As Claxton et al. only included changes in health spending in the first year, while estimating lifetime health benefits, all future costs were missing from their estimates. Consequently, we aimed to update their estimates to account for future costs:

$k^{\dagger}=k+\frac{\Delta L \times\left(c_{\mathrm{r}}+c_{\mathrm{u}}\right)}{\Delta \mathrm{QALYs}}$

where $k$ denotes the Claxton et al. threshold estimate and $k^{\dagger}$ is the threshold including future costs of both related and unrelated diseases. The life-table calculations for estimating future healthcare costs were carried out for each separate PBC, where future costs are derived from previous work by Perry-Duxbury et al. [18]. The increase in total health spending is distributed over the different PBCs using the expenditure elasticities. The disease-specific impacts of this increase, in comparison to a counterfactual in which the budget did not increase, on cause-specific mortality (used in the cause-deleted life tables to estimate LYs gained) were calculated using the outcome elasticities estimated by Claxton et al. Note that outcome elasticities were not available for all disease categories, mostly because of little to no mortality effects in the specific PBC. For these PBCs, future healthcare costs do not impact the threshold. To translate estimates expressed in pounds per $L Y$ gained to pounds per $Q A L Y$ gained, we used the $\mathrm{PBC}$-specific ratios of incremental costs per LY to costs per QALY from Claxton et al., who used PBC-specific quality-of-life decrements by age and sex. We translate LYs to QALYs using the aforementioned ratios (Table 1).

Overall threshold estimates were calculated using weights based on the percentage share of health effects provided by
Table 1 Ratios (cost per LY/cost per QALY) from Claxton et al. used to translate LYs, using disease-specific quality of life

\begin{tabular}{lrrr}
\hline & Cost per LY & Cost per QALY & $\begin{array}{l}\text { Cost per LY/ } \\
\text { costs per } \\
\text { QALY }\end{array}$ \\
\hline Infectious diseases & 61,425 & 20,829 & 2.95 \\
Cancer & 11,931 & 16,997 & 0.70 \\
Endocrine & 38,122 & 3124 & 12.20 \\
Neurological & 92,282 & 5480 & 16.84 \\
Circulatory & 6544 & 7038 & 0.93 \\
Respiratory & 28,528 & 1998 & 14.28 \\
Gastrointestinal & 12,983 & 7293 & 1.78 \\
Genitourinary & 141,746 & 43,813 & 3.24 \\
Maternity and neo- & $1,608,817$ & $2,969,208$ & 0.54 \\
nates & & & \\
\hline
\end{tabular}

Only for disease categories for which there is an observed mortality effect

$L Y$ life-year, $Q A L Y$ quality-adjusted life-year

Claxton et al. The equations and further explanation of the life-tables approach are presented below.

\subsubsection{Estimating Future Costs and LYs}

In both the situations in which the budget increases for PBC, which includes health for related diseases $r$ and the counterfactual, the lifetime expected discounted costs and LYs, $L_{\mathrm{r}}(a)$ can be estimated using the formulas below.

$C_{\mathrm{r}}(a=b)=\sum_{i=b}^{100}\left(1.035^{i-b} \times c(a=i) \times \prod_{j=b}^{i} e^{-m_{\mathrm{r}}(a=j)-m_{\mathrm{u}}(a=j)}\right)$

$L_{\mathrm{r}}(a=b)=\sum_{i=b}^{100}\left(1.035^{i-b} \times \prod_{j=b}^{i} e^{-m_{\mathrm{r}}(a=j)-m_{\mathrm{u}}(a=j)}\right)$

where $C_{\mathrm{r}}(a)$ represents remaining lifetime healthcare costs for someone at a particular age (here age is denoted $a$ that takes on value $b$ in Eqs. (5) and (6) treated in PBCs with related diseases $r$ and $L_{\mathrm{r}}(a)$ represents remaining LYs at a particular age treated in PBCs with related diseases. $r .1 .035$ refers to the discount rate for costs in the UK, 3.5\%.c(a) represents yearly per capita costs by age and $m_{\mathrm{r}}(a)$ and $m_{\mathrm{u}}(a)$ represent related and unrelated mortality rates by age, where $a$ is a single year of age from which costs are aggregated.

We assume that yearly per capita costs depend on age and time to death.

$c(a)=\left[m_{\mathrm{r}}(a)+m_{\mathrm{u}}(a)\right] \times c_{\mathrm{d}}(a)+\left[1-m_{\mathrm{r}}(a)-m_{\mathrm{u}}(a)\right] \times c_{\mathrm{s}}(a)$

Here, $c_{\mathrm{d}}(a)$ denotes yearly costs at age $a$ conditional on dying at age $a$ (average costs in the last year of life) while 
$c_{\mathrm{s}}(\mathrm{a})$ denotes yearly costs for those who do not die at age $a$. These are derived from previous work by Perry-Duxbury et al. (2020). Note that we do not make a distinction between related and unrelated future costs as neither was included in the Claxton et al. study. When expenditure increases, related mortality rates, $m_{\mathrm{r}}(a)$, are multiplied by outcome elasticities (which are negative) and 1.01, reflecting the $1 \%$ increase in expenditure (Eq. 8) [7]. The lifetime costs and LYs from the budget increase, $C_{\mathrm{r}}^{\prime}(a)$ and $L_{\mathrm{r}}^{\prime}(a)$, are calculated by replacing disease-related mortality with $m_{\mathrm{r}}{ }^{\prime}(a)$, where ${ }^{\prime}$ indicates the situation in which expenditure increases. To be consistent with Claxton et al., we assume a 1-year change in mortality.

$m_{\mathrm{r}}^{\prime}(a)=m_{\mathrm{r}}(a) \times[1+0.01 \times o(r)]$

where $o(r)$ represents the outcome (mortality) elasticity for PBC containing spending for disease $r$.

\subsubsection{Estimating Disease-Specific and NHS-Wide Thresholds}

We use PBC-specific ratios of costs per LY to costs per QALY gained from Claxton et al. to translate costs per LY gained to costs per QALY gained. Thus, QALYs for the situation in which expenditure increases and the counterfactual (denoted $Q_{\mathrm{r}}{ }^{\prime}(a)$ and $Q_{\mathrm{r}}(a)$ ) are calculated by multiplying the respective remaining LYs by these ratios. We then estimate both PBC-specific thresholds, $k(r)^{\dagger}$, and a subsequent threshold for all NHS spending, $k^{\dagger}$, which is arguably the most policy-relevant estimate. and LYs gained within a PBC, which are provided in Table 1. Programme budgeting categories for neurological and endocrine disorders have relatively high ratios of cost per LY to costs per QALY, meaning that an increase in the healthcare budget leads to more quality-of-life gains than LY gains. The opposite is seen in diseases such as cancer and circulatory disease where most health gains are due to increases in length of life. A second reason is that the older the patient population within a PBC, the more future costs they will incur. This is because of medical consumption increasing with age and discounting - the higher the age at which one receives an intervention, the less the corresponding future costs are discounted. Thus, it is of little surprise that diseases such as cancer and circulatory disease have higher adjusted thresholds, while diseases that tend to affect the young, such as infectious diseases, only see future healthcare cost additions of $£ 510$.

The final row of Table 2 shows the difference in the costeffectiveness threshold when calculated for the full NHS budget-a $1 \%$ increase in the NHS budget divided by the sum of all LYs or QALYs. This difference is a weighted average of the PBC-specific threshold estimates, leading to an addition of $£ 743$ to the overall threshold. The impact of future healthcare costs on the overall threshold estimate is limited because much of the share of total health effects is attributed to respiratory disease (30\%), neurological disease (14\%) and circulatory disease (14\%), with only the latter of the three PBCs having high future costs per QALY. Additionally, some shares of total health effects are attributed to PBCs with no mortality effects, such as mental health (12\%).

$k(r)^{\dagger}=k(r)+\frac{\sum_{j=0}^{100} \operatorname{pop}(a=j) \times\left[\left(C_{r}^{\prime}(a=j)-C_{\mathrm{r}}(a=j)\right) \times p(a=j, r)\right]}{\sum_{i=0}^{100} \operatorname{pop}(a=i) \times\left[\left(Q_{r}^{\prime}(a=i)-Q_{\mathrm{r}}(a=i)\right) \times p(a=i, r)\right]}$

$k^{\dagger}=\sum_{r \in D}\left(k(r)^{\dagger} \times w(r)\right)$

In the above equations, $k$ represents the Claxton et al. threshold estimate while $k^{\dagger}$ represents threshold estimates including future costs. $\operatorname{pop}(a)$ is the population size of age group $a, p(a, r)$ is the percentage of patients in age group $a$ treated in PBC with related diseases $r, D$ represents the set of all PBCs and $w(r)$ represents the weighting taken as a percentage share of total health effects for a PBC with related diseases $r$ [7].

\section{Results}

The impact of including future costs on PBC-specific threshold estimates varies considerably. For example, neurological and endocrine diseases sit at the low end of the spectrum, while cancer has far more future healthcare costs attributed to it. The first reason for this is the difference between QALYs

\section{Discussion}

We find that the impact of including future costs on threshold estimates is limited, which is due to the fact in the Claxton et al. paper that the marginal returns to health spending were, to a large extent, driven by improvements in quality of life as opposed to increases in length of life. Given that ICERs for specific interventions might be affected more heavily by the inclusion of future costs, these findings have important implications for decisions based on cost effectiveness. This implies that interventions that are life improving rather than life extending and interventions in younger patients will be more likely to be reimbursed as compared to when analyses unadjusted for future costs are conducted. For instance, in our earlier work [18], we found that including future costs in three economic evaluation cases led to increases in the ICERs of between $£ 3200$ and $£ 7200$. These 
Table 2 Addition of future costs to PBC-specific and overall threshold estimates (£)

\begin{tabular}{|c|c|c|c|c|c|c|c|}
\hline \multicolumn{4}{|l|}{ A. Cost per LY } & \multicolumn{4}{|c|}{ B. Cost per QALY (PBC-specific QoL $^{\mathrm{a}}$ ) } \\
\hline & Original estimate & $\begin{array}{l}\text { Addition of } \\
\text { future costs }\end{array}$ & Final estimate & & Original estimate & $\begin{array}{l}\text { Addition of } \\
\text { future costs }\end{array}$ & Final estimate \\
\hline Infectious diseases & 61,425 & 1502 & 62,927 & Infectious diseases & 20,829 & 510 & 21,339 \\
\hline Cancer & 11,931 & 2508 & 14,439 & Cancer & 16,997 & 3574 & 20,571 \\
\hline Blood & - & - & 0 & Blood & 9419 & - & 9419 \\
\hline Endocrine & 38,122 & 2205 & 40,327 & Endocrine & 3124 & 181 & 3305 \\
\hline Mental health & - & - & 0 & Mental health & 18,744 & - & 18,744 \\
\hline Neurological & 92,282 & 1816 & 94,098 & Neurological & 5480 & 108 & 5588 \\
\hline Vision & - & - & 0 & Vision & 45,788 & - & 45,788 \\
\hline Hearing & - & - & 0 & Hearing & 6239 & - & 6239 \\
\hline Circulatory & 6544 & 2957 & 9501 & Circulatory & 7038 & 3181 & 10,219 \\
\hline Respiratory & 28,528 & 2711 & 31,239 & Respiratory & 1998 & 190 & 2188 \\
\hline Gastrointestinal & 12,983 & 2111 & 15,094 & Gastrointestinal & 7293 & 1186 & 8479 \\
\hline Skin & - & - & 0 & Skin & 101,042 & - & 101,042 \\
\hline Musculoskeletal & - & - & 0 & Musculoskeletal & 15,628 & - & 15,628 \\
\hline Genitourinary & 141,746 & 3058 & 144,804 & Genitourinary & 43,813 & 945 & 44,758 \\
\hline $\begin{array}{l}\text { Maternity and neo- } \\
\text { nates }\end{array}$ & $1,608,817$ & 953 & $1,609,914$ & $\begin{array}{l}\text { Maternity and neo- } \\
\text { nates }\end{array}$ & $2,969,208$ & 1758 & $2,970,966$ \\
\hline Total $^{\mathrm{b}}$ & 25,214 & 2673 & 28,403 & Total $^{\mathrm{b}}$ & 12,936 & 743 & 13,679 \\
\hline
\end{tabular}

Costs and outcomes are both discounted by $3.5 \%$ as recommended by NICE guidelines

$L Y$ life-year, $P B C$ programme budgeting category, $Q A L Y$ quality-adjusted life-year, $Q o L$ quality of life

${ }^{\mathrm{a}} \mathrm{QoL}$ adjusted for $\mathrm{PBC}$-specific decrements by age and sex

${ }^{b}$ Percentage share of total health effects is used as the weighting for all total estimates. Table A uses a share of change in net years of life lost, Table B uses a share of change in disease-specific QALYs

increases in ICERs are much greater than the increase in the threshold.

We estimate adjusted PBC-specific thresholds as well as an adjusted overall threshold. The updated overall threshold was $£ 13,679$ per QALY, $£ 743$ higher than the original estimate of $£ 12,936$. Programme budgeting category-specific estimates of the adjustment ranged from $£ 108$ per QALY to $£ 3574$ per QALY when using disease-specific and agespecific quality-of-life estimates. Programme budgeting categories with the highest threshold varied depending on the denominator of the estimate. For example, the PBC with the largest addition to the threshold was genitourinary disease (£3058) when dividing costs by LYs gained and cancer (£3574) when dividing by QALYs. These differences are solely because of the ratios between cost per LY and cost per QALY taken from Claxton et al., some of which are surprising. For example, regarding respiratory diseases, the ratio suggests that, given a $1 \%$ increase in the budget, increases in QALYs are 14-fold the increases in LYs. It seems worthwhile for future studies to further investigate these influential relationships.

The overall threshold is arguably the most relevant estimate for decision makers, as resource allocation decisions in the NHS are made centrally across the entire healthcare budget rather than on a per disease basis [20]. ${ }^{1}$ The overall adjusted threshold we estimate $(£ 13,679)$ is higher than that of the unadjusted threshold estimate $(£ 12,936)$ [Claxton et al.] but substantially lower than the threshold range used in practice by NICE [3].

There are limitations to our approach. First, in this demonstrative case study, we only update the point estimate of the empirical NHS threshold. This is because most of the inputs for our calculations, such as population health, disease prevalence and mortality, are stable. Possible sources of uncertainty are the (disease-specific) outcome elasticities estimated by Claxton et al.; however, investigating this goes beyond the scope of our paper. Second, we were only able to update the PBC-specific thresholds for PBCs for which mortality elasticities were available from the existing empirical work, and thus our overall threshold estimate may be an underestimation if the increase in expenditure did indeed affect the mortality of PBCs for which elasticities were not available. That being said, the majority of QALYs gained

\footnotetext{
${ }^{1}$ An exception to this rule may be the NHS Cancer Drugs Fund, which pays for cancer drugs that would otherwise be rejected by NICE for being too expensive.
} 
from the excluded PBCs were due to increases in quality of life, thus the effect is likely to be modest. Finally, we do not separate the impact of the increase in health spending on future costs into impacts on unrelated and related costs. If an increase in the budget turns out to increase the quality of life and/or lead to savings in related costs, then our adjusted threshold value would be an overestimate.

It is important to note that this paper solely addresses the assumption that future costs can be excluded from the supply-side threshold, and not the other assumptions used in the work by Claxton et al. Others have argued that referring to this threshold estimate as the opportunity cost is inaccurate unless the current bundle of interventions being used is optimal and the least cost-effective intervention is removed from the bundle when a new intervention enters [21]. These are important discussions to have, especially if NICE, or other health technology assessment bodies, are to start using a supply-side threshold for reimbursement decisions. Future work on assumptions regarding how and where displacement occurs can only move us closer to the best operationalised threshold estimate.

\section{Conclusions}

As NICE and other health technology assessment bodies around the world review the methods for conducting economic evaluations, we feel it is time for them to consider using an empirically estimated supply-side threshold and including future healthcare costs both in cost-effectiveness analyses as well as in the threshold that the results of these analyses are judged against. Such an approach will help to fully capture the threshold associated with new health technologies and to maximise health gains that can be achieved from any given level of health spend, particularly relevant at a time when health budgets are increasingly stretched by the coronavirus pandemic.

Acknowledgements We thank Prof. Werner Brouwer (Erasmus University Rotterdam) for his helpful comments on a draft of this manuscript. We thank Prof. Karl Claxton (University of York) for his comments on the manuscript and its empirical and theoretical underpinnings. We also thank all those who participated in the discussion of the earliest iteration of this paper at the 2019 Winter Health Economists' Study Group.

\section{Declarations}

Funding The authors received no specific funding for this work.

Conflicts of interest/competing interests The authors declare no conflicts of interest.
Ethics approval This study uses aggregate data that has already been collected and published online. No ethical approval is required.

Consent to participate This is not applicable as no individuals participated in this research.

Consent for publication This is not applicable as no individuals participated in this research.

Availability of data and material Data were taken from published works (Perry-Duxbury et al. 2020, Claxton et al. 2015) and the PAID UK tool (https://www.imta.nl/paid/). Data used in Perry-Duxbury et al. were open aggregate data.

Code availability Code available upon request.

Author contributions Conceptualization: MP-D, JL, MA, PvB. Methodology: MP-D, JL, MA, PvB. Formal analysis and investigation: MP-D. Writing, original draft preparation: MP-D. Writing, review and editing: MP-D, JL, MA, PvB. Funding acquisition: not applicable. Resources: JL. Supervision: PvB.

Open Access This article is licensed under a Creative Commons Attribution-NonCommercial 4.0 International License, which permits any non-commercial use, sharing, adaptation, distribution and reproduction in any medium or format, as long as you give appropriate credit to the original author(s) and the source, provide a link to the Creative Commons licence, and indicate if changes were made. The images or other third party material in this article are included in the article's Creative Commons licence, unless indicated otherwise in a credit line to the material. If material is not included in the article's Creative Commons licence and your intended use is not permitted by statutory regulation or exceeds the permitted use, you will need to obtain permission directly from the copyright holder. To view a copy of this licence, visit http://creativecommons.org/licenses/by-nc/4.0/.

\section{References}

1. Brouwer W, van Baal P, van Exel J, Versteegh M. When is it too expensive? Cost-effectiveness thresholds and health care decisionmaking. Eur J Health Econ. 2019; 20:175-80. https://doi.org/10. 1007/s10198-018-1000-4.

2. Schwarzer R, Rochau U, Saverno K, Jahn B, Bornschein B, Muehlberger N, et al. Systematic overview of cost-effectiveness thresholds in ten countries across four continents. J Comp Eff Res. 2015;4(5):485-504. https://doi.org/10.2217/cer.15.38.

3. Uk NICE. Guide to the methods of technology appraisal. London: National Institute for Health and Clinical Excellence; 2013.

4. Appleby J, Devlin N, Parkin D, Buxton M, Chalkidou K. Searching for cost effectiveness thresholds in the NHS. Health Policy. 2009;91(3):239-45. https://doi.org/10.1016/j.healthpol.2008.12. 010.

5. van Baal P, Perry-Duxbury M, Bakx P, Versteegh M, van Doorslaer E, Brouwer W. A cost-effectiveness threshold based on the marginal returns of cardiovascular hospital spending. Health Econ. 2019;28(1):87-100. https://doi.org/10.1002/hec.3831.

6. Vallejo-Torres L, García-Lorenzo B, Castilla I, Valcárcel-Nazco C, García-Pérez L, Linertová R, et al. On the estimation of the cost-effectiveness threshold: why, what, how? Value Health. 2016;19(5):558-66. https://doi.org/10.1016/j.jval.2016.02.020.

7. Claxton K, Martin S, Soares M, Rice N, Spackman E, Hinde S, Devlin N, Smith PC, Sculpher M. Methods for the estimation of 
the National Institute for Health and Care excellence cost-effectiveness threshold. Health Technol Assess. 2015;19(14). https:// doi.org/10.3310/hta19140.

8. Martin S, Rice N, Smith PC. Comparing costs and outcomes across programmes of health care. Health Econ. 2012;21(3):31637. https://doi.org/10.1002/hec.1716.

9. Martin S, Rice N, Smith PC. Does health care spending improve health outcomes? Evidence from English programme budgeting data. J Health Econ. 2008;27(4):826-42. https://doi.org/10.1002/ hec. 1716.

10. Meltzer D. Accounting for future costs in medical cost-effectiveness analysis. J Health Econ. 1997;16:33-64.

11. Rappange DR, Van Baal PHM, Van Exel NJA, Feenstra TL, Rutten FFH, Brouwer WBF. Unrelated medical costs in life-years gained: should they be included in economic evaluations of healthcare interventions? Pharmacoeconomics. 2008;26(10):81530. https://doi.org/10.2165/00019053-200826100-00003.

12. van Baal P, Meltzer D, Brouwer W. Future costs, fixed healthcare budgets, and the decision rules of cost-effectiveness analysis. Health Econ. 2016;25(2):237-48. https://doi.org/10.1002/hec. 3138.

13. Morton A, Adler AI, Bell D, Briggs A, Brouwer W, Claxton K, Craig N, Fischer A, McGregor P, van Baal P. Unrelated future costs and unrelated future benefits: reflections on NICE guide to the methods of technology appraisal. Health Econ. 2016;25:9338. https://doi.org/10.1002/hec.3366.

14. van Baal P, Morton A, Brouwer W, Meltzer D, Davis S. Should cost effectiveness analyses for NICE always consider future unrelated medical costs? BMJ. 2017;359:j5096. https://doi.org/10. 1136/bmj.j5096.
15. de Vries LM, van Baal PHM, Brouwer WBF. Future costs in costeffectiveness analyses: past, present, future. Pharmacoeconomics. 2019;37(2):119-30. https://doi.org/10.1007/s40273-018-0749-8.

16. Barnsley P, Towse A, Sussex J. Critique of CHE research paper 81: methods for the estimation of the NICE cost effectiveness threshold. Office Health Econ. London; 2013. https://doi.org/10. 2139/ssrn.2637927.

17. Raftery J. NICE's cost-effectiveness range: should it be lowered? Pharmacoeconomics. 2014;32:613-5. https://doi.org/10.1007/ s40273-014-0158-6.

18. Perry-Duxbury M, Asaria M, Lomas J, van Baal P. Cured today, ill tomorrow: including future unrelated medical costs in economic evaluations for England \& Wales. Value Health. 2020;23(8):102733. https://doi.org/10.1016/j.jval.2020.05.006.

19. Kellerborg K, Perry-Duxbury M, de Vries L, van Baal P. Practical guidance for including future costs in economic evaluations in the Netherlands: introducing and applying PAID 3.0. Value Health. 2020;23(11):1453-61. https://doi.org/10.1016/j.jval.2020.07.004.

20. Maynard A, Bloor K. The economics of the NHS cancer drugs fund. Appl Health Econ Health Policy. 2011. https://doi.org/10. 2165/11585750-000000000-00000.

21. Eckermann S, Pekarsky B. Can the real opportunity cost stand up: displaced services, the straw man outside the room. Pharmacoeconomics. 2014;32(4):319-25. https://doi.org/10.1007/ s40273-014-0140-3. 